\title{
EDITORIAL
}

\section{Big in Japan}

As the gravitational wave detector KAGRA goes online, we look at the future of big science projects in Japan.

Last month, KAGRA, a gravitational-wave detector located in Kamioka, Japan, was ramping up to begin observations. KAGRA achieves several firsts. It is the first gravitational wave detector in Asia. It is the first underground detector, with its two $3 \mathrm{~km}$ arms located in tunnels $200 \mathrm{~m}$ under Mount Ikenoyama, and the first to use cryogenically cooled mirrors.

"The gravitational-wave community has been waiting with great anticipation for KAGRA to come online. KAGRA uses advanced cryogenic mirror technologies that go beyond the Advanced LIGO and Advanced Virgo interferometers," said David Reitze, Executive Director of LIGO. The $¥ 16.4$ billion (US $\$ 148$ million) detector is one of the big science projects listed in the master plan announced on 30 January by the Science Council of Japan. The country is committing to a number of exciting large-scale science projects.

KAGRA's location at Kamioka is not coincidental. Takaaki Kajita, recipient of the 2015 Nobel prize in Physics for the neutrino experiments he conducted at the Kamioka Observatory, is one of the driving forces behind KAGRA. He is also currently the principal investigator of the project. KAGRA got funding 5 years before gravitational waves were first detected, and the excavation of the tunnels began in 2012. Initial progress was rapid: the two $3 \mathrm{~km}$ tunnels and the central and end stations were completed in just 2 years. But then, the tunnels flooded because Mount Ikenoyama is an aquifer - which comes in handy for neutrino experiments that use tanks filled with tonnes of water. However, it is not great for keeping a tunnel dry, so the KAGRA team had to design a drainage system that can suck up to 1,000 tonnes of water per hour in the wettest

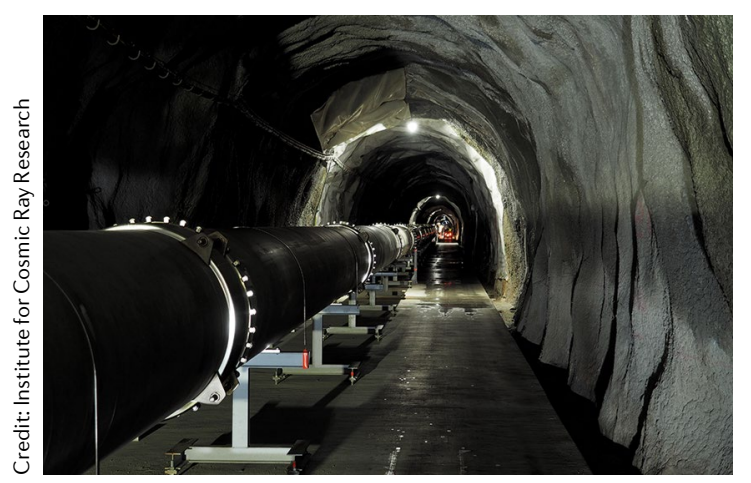

season. This was not the only challenge: the KAGRA scientists had to find solutions to a number of unique problems such as installing high-vacuum equipment in dirty, high-humidity underground conditions, and designing and testing the cryogenic cooling system for the mirrors - a technology that has never been used in gravitational wave detectors before. By working with dozens of specialized Japanese companies the KAGRA team was able to overcome these obstacles in record time.

In addition to KAGRA, the Science Council of Japan selected 31 projects in its high-priority list. These include the Super-B factory at the High Energy Accelerator Research Organization (KEK) - the next generation of the KEKB electron-positron collider used for the study of B mesons and an upgrade of the J-PARC facility to enable fundamental and applied studies using high-energy proton beams. On the list there is also the LiteBIRD, a space mission for studies of B-mode polarization and inflation from the cosmic background radiation, and an investment in the Subaru 2 and ALMA2 telescopes. Another project is ALFA, an attosecond laser light user facility.

Last month, another item was added to this list of ambitious projects: the funding was approved for Hyper-Kamiokande, the scale-up of the existing Super-Kamiokande neutrino detector located at the Kamioka Observatory. The project is estimated to cost $¥ 64.9$ billion (US\$600 million) and together with the J-PARC upgrade is expected to boost precision measurements such as searches for $\mathrm{CP}$ violation discussed in a Feature in this issue.

As all funding decisions go, there is rejoicing but also disappointment, most notably in this case, the proposed International Linear Collider, a future $250 \mathrm{GeV}$ linear accelerator (see the Lab news piece in this issue), not making it to the Science Council of Japan list. However, the $¥ 800$ billion ( $\$$ US 7.5 billion) future accelerator project may still go ahead, the Japanese government likely waiting to see the outcome of the European Strategy for Particle Physics, which is due to be updated by May this year, before committing to contribute to it.

Big science projects call for substantial research and development efforts and require advanced technologies and manufacturing that can often be found in industry. Considering that, Japan's commitment to invest in such projects plays well on the country's strengths. 